\title{
Reconstruction of the Proton Source in Relativistic Heavy Ion Collisions
}

\author{
Alberto Polleri $^{a}{ }^{*}$, Raffaele Mattiello $^{a}$, Igor N. Mishustin ${ }^{a, b, c}$ and Jakob P. Bondorf ${ }^{a}$. \\ aThe Niels Bohr Institute, Blegdamsvej 17, DK-2100 Copenhagen Ø, Denmark. \\ bThe Kurchatov Institute, Russian Scientific Center, Moscow 123182, Russia. \\ 'Institute for Theoretical Physics, J.W.Goethe-University, 60054 Frankfurt, Germany \\ We describe a direct method to reconstruct the transverse proton source formed in a rel- \\ ativistic heavy ion collision, making use of experimentally measured proton and deuteron \\ spectra and assuming that deuterons are formed via two-nucleon coalescence. We show \\ that an ambiguity with respect to the source temperature still persists and we indicate a \\ possible solution to the problem.
}

It has been recently shown that a simple description of the proton phase space distribution can provide a good qualitative understanding of deuteron spectra [1]. On the other hand, it appeared clear that a more solid method to extract the properties of the source had to be established. We have therefore reconstructed the phase space distribution of protons directly from the observed proton and deuteron spectra, exploiting the coalescence prescription together with the notion of collective flow. Here we will outline the procedure while all details can be found in [2]. We make use of a relativistic description of collective flow based on the boost-invariant picture for the longitudinal expansion, together with a longitudinally-independent transverse velocity, and therefore write the proton phase space distribution as

$f_{p}(x, p)=(2 \pi)^{3} \exp \left(-p_{\mu} u^{\mu}(x) / T_{0}\right) B_{p} n_{p}\left(r_{\perp}\right)$,

where $p_{\mu} u^{\mu}(x)=\gamma(r)\left(m_{\perp} \cosh (y-\eta)-\vec{p}_{\perp} \cdot \vec{v}(\vec{r})\right)$ is the energy in the global frame and $B_{p}$ is the normalisation coefficient of the Boltzmann distribution in the local frame. The local density $n_{p}(r)$ is assumed to be independent of the longitudinal rapidity.

The deuteron phase space distribution is calculated using the coalescence model. Its evaluation is simplified when considering large and hot systems, neglecting the smearing effect of the deuteron Wigner density in comparison to the characteristic scales of the system in position and momentum space. One therefore obtains the deuteron phase space distribution

$f_{d}(x, p) \simeq \frac{3}{8} R_{n p}\left[f_{p}(x, p / 2)\right]^{2}$.

The neutron to proton ratio in the source was taken to be $R_{n p}=1.2$. The deuteron phase space distribution has the same structure as the proton one in eq. (1), now with $n_{p}(r)$ replaced by $n_{d}(r)=\lambda_{d} n_{p}^{2}(r)$, where $\lambda_{d}=3 / 8 R_{n p}(2 \pi)^{3} B_{p}^{2} / B_{d}$.

${ }^{*}$ Present address: Institute for Theoretical Physics, Philosophenweg 19, 69120 Heidelberg, Germany. 
One can now calculate proton and deuteron invariant momentum spectra using the Cooper-Frye formula, on an approximate freeze-out hypersurface of constant longitudinal proper time $\tau_{0}$. The integrations over the space-time rapidity and the azimuthal angle can be easily performed, yielding an expression in terms of the two functions $v(r)$ and $n(r)$. The ambiguity in the description of the single particle spectrum is explicit, since the two functions cannot be mapped out uniquely from only one function as the transverse momentum spectrum. To partially remove the ambiguity, we first change the integration variable and introduce the auxiliary function $\tilde{n}$ through the relation $v d v \tilde{n}(v)=r d r \tau_{0} n(r)$, obtaining the new expression for the momentum spectrum

$S\left(p_{\perp}\right)=4 \pi B m_{\perp} \int_{0}^{1} d v v K_{1}\left(\frac{\gamma m_{\perp}}{T_{0}}\right) I_{0}\left(\frac{v \gamma p_{\perp}}{T_{0}}\right) \tilde{n}(v)$.

The one-to-one correspondence between $\tilde{n}(v)$ and $S\left(p_{\perp}\right)$ is now evident. We then make use of the coalescence model and the definition of $\tilde{n}$, both for protons and for deuterons, to obtain a first order differential equation that can be directly integrated and gives the closed solution

$r^{2}=2 \frac{\lambda_{d}}{\tau_{0}} \int_{0}^{v} d u u \frac{\tilde{n}_{p}^{2}(u)}{\tilde{n}_{d}(u)}$.

Therefore, by independently extracting the functions $\tilde{n}_{p}$ and $\tilde{n}_{d}$ from the observed momentum spectra $S_{p}\left(p_{\perp}\right)$ and $S_{d}\left(p_{\perp}\right)$, we can find the function $r(v)$ by a simple numerical integration. Inverting the obtained function as $r(v) \rightarrow v(r)$, we obtain the collective velocity profile. We also obtain the local proton density as

$n_{p}(r)=\frac{v(r)}{r} \frac{d v(r)}{d r} \frac{\tilde{n}_{p}(v(r))}{\tau_{0}}$.

The described procedure was applied to the transverse momentum spectra, resulting from $\mathrm{Pb}+\mathrm{Pb}$ collisions and measured by the NA44 collaboration at the CERN-SPS [3] . We fitted $\tilde{n}_{p}$ and $\tilde{n}_{d}$ to these data, using eq. (3) for protons and deuterons and assuming different values for $T_{0}$, from $50 \mathrm{MeV}$ to $150 \mathrm{MeV}$. We chose a form of the profile functions characterized by three parameters, and we extracted their value from the experimental data with a Monte Carlo search minimising $\chi^{2}$. The fitted spectra are shown, for the extreme values of temperature considered, in the top part of Fig. 1. Although evaluated at different temperatures, they are indistinguishable from one another. On the other hand, the profiles $\tilde{n}$ result to be very different for different temperatures.

In all calculations the freeze-out time was fixed at $\tau_{0}=10 \mathrm{fm} / \mathrm{c}$ [四]. After numerical integration of eq. (4), we obtained the function $v(r)$ shown in Fig. 2 for the two extreme temperatures. It shows a linear rise at small $r$ and saturates for large $r$. The velocity profiles clearly depend on the temperature chosen. The local proton density is also plotted in Fig. 2. At high temperature the density shows a shell-like structure which disappears as the temperature is lower. Similar shell-like structures have been recently found in analytic solutions of non-relativistic hydrodynamics [5]. From the plots one can observe the different transverse sizes corresponding to different temperatures. It is therefore necessary to know $T_{0}$ precisely in order to determine the system size. This information cannot be extracted solely from proton and deuteron spectra. Contrary to what is commonly done, 


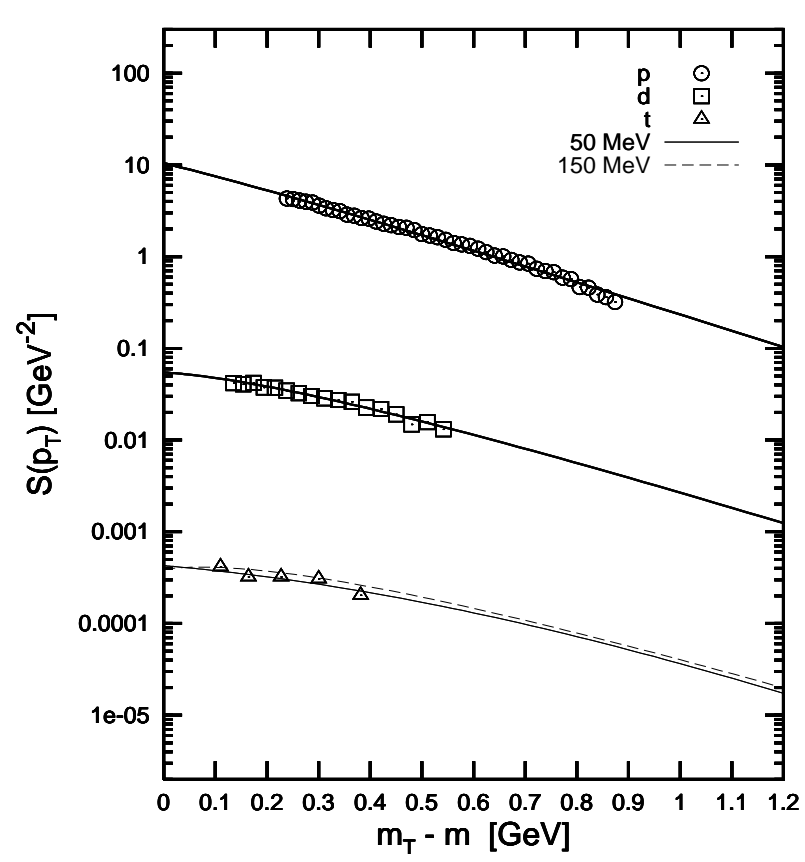

Figure 1. Comparison of the transverse spectra of $p, d$ and $t$, calculated within the improved coalescence model, with the measurements obtained by the NA44 experiment [3].

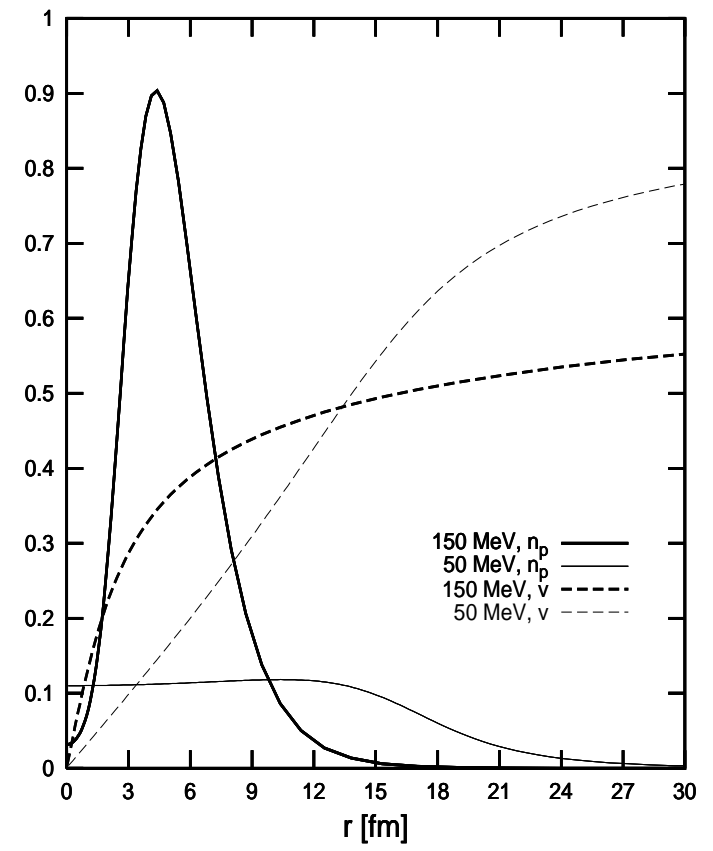

Figure 2. Reconstructed local density and flow of protons as function of the transverse radius, for the extreme values of $T_{0}$. $n_{p}$ is rescaled to be compared with $v$.

source radii cannot be extracted from the $d / p^{2}$ ratio ( $B_{2} \sim$ emitting volume) unless $T_{0}$ is known.

It is clear that to resolve the remaining ambiguity in the source temperature one needs some additional experimental information. Recently, $\pi \pi$ correlation data were used as a constraint [6]. Since pions may freeze-out in a different way than protons, it would be even better to consider $p p$ correlations, although they are more sensitive to final state interactions than pions. On the other hand, heavier clusters can provide additional constraints. We now address this issue following [7] and we describe the fusion process of $A$-nucleons into a bound state within the density matrix formalism. Making use of the same approximation leading to eq. (2), one obtains cluster phase space distribution

$f_{c}(x, p) \simeq g_{A} \frac{\left(R_{n p}\right)^{N}}{Z ! N !}\left[f_{p}(x, p / A)\right]^{A}$.

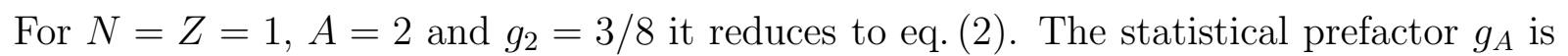
of crucial importance. In the conventional approach we have $g_{A}=\left(2 S_{A}+1\right) Z ! N ! / 2^{A} A$ !, which results in $g_{d}=3 / 8, g_{t}=g^{3} \mathrm{He}=1 / 12$, and $g_{4} \mathrm{He}=1 / 96$. Although straightforward, this approach has been successful only for the description of deuteron production. In fact, it significantly underestimates the yields of heavier clusters. A possible improvement can be achieved by allowing for additional formation processes. Besides the already considered 
direct process, it is possible that deuteron-like correlations contribute to cluster formation. For the triton, as an example, we should therefore account for the possibility that a proton and a neutron are already in a bound state with deuteron quantum numbers and coalesce with another neutron, with a different statistical prefactor. More precisely we can write $g_{t}=g_{p n n \rightarrow t}+2 g_{p n \rightarrow d} g_{d n \rightarrow t}$, where the factor 2 counts the different ways to associate the proton with the two neutrons in forming a deuteron. The spin-isospin counting is straightforward and gives the modified statistical prefactor $g_{t}=1 / 3$, therefore enhancing the triton yield by a factor 4 . The same arguments apply to ${ }^{3} \mathrm{He}$, so that $g^{3} \mathrm{He}=1 / 3$. The case of ${ }^{4} \mathrm{He}$ is more involved. Counting all the possible processes we obtained $g^{4} \mathrm{He}=13 / 48$, so that the ${ }^{4} \mathrm{He}$ yield is increased by a factor 26 . For more details see [8].

We now use the coalescence model in this improved version to calculate the transverse mass spectrum of tritons. Using the flow and density profiles extracted from the analysis of $p$ and $d$ spectra, we examine to what extent heavier clusters can constrain the ambiguity of the temperature. Using eqs. (1) and (6), we obtain $n_{t}(r)=\lambda_{t} n_{p}^{3}(r)$, with $\lambda_{t}=g_{t}\left(R_{n p}\right)^{2} / 2(2 \pi)^{6} B_{p}^{3} / B_{t}$, while the collective velocity is the same for all clusters. The triton spectrum is plotted in Fig. 1, together with the previously fitted $p$ and $d$ spectra. The absolute values and the shape compare very well with the experimental data. This confirms, that the improved statistical approach is consistent with the measured spectra. Furthermore, the results obtained with the two extreme temperatures show a different curvature. The high temperature case presents a clear bending over, absent for low temperature. We argue that this specific difference might narrow down the allowed temperatures and therefore provide additional constraints. Unfortunately, the triton spectrum was measured only in a limited range in transverse momentum, and therefore a quantitative fit is not useful.

We also suggest that a further possibility to constrain the temperature lies in the combined study of single and composite spectra, together with $p p$ correlations. The extracted profiles shown in Fig. 2 could in fact be used to evaluate the $p p$ correlation function, which is sensitive to temperature and flow in a different way respect to inverse slopes of spectra. These remarks are important for the interpretation of the future experiments at the Relativistic Heavy Ion Collider.

\section{REFERENCES}

1. A. Polleri, J.P. Bondorf and I.N. Mishustin, Phys. Lett. B 419, 19 (1998).

2. A. Polleri, R. Mattiello, I.N. Mishustin and J.P. Bondorf, nucl-th/9904012.

3. I. Bearden and A.G. Hansen for the NA44 Collaboration, These Proceedings.

4. H.W. Barz, J.P. Bondorf, J.J. Gaardhoje and H. Heiselberg, Phys. Rev. C56, 1553 (1997).

5. T. Csörgő, nucl-th/9809011.

6. R. Scheibl and U. Heinz, Phys. Rev. C59, 1585 (1998).

7. J.L. Nagle, B.S. Kumar, D. Kusnezov, H. Sorge and R. Mattiello, Phys. Rev. C 53, 367 (1996), R. Mattiello, H. Sorge, H. Stöcker and W. Greiner, Phys. Rev. C 55, 1443 (1997).

8. A. Polleri, Ph.D. Thesis, University of Copenhagen (1999). 